\title{
Erratum: Relationship between bond-breakage correlations and four-point correlations in heterogeneous glassy dynamics: Configuration changes and vibration modes [Phys. Rev. E 86, 041504 (2012)]
}

Hayato Shiba, Takeshi Kawasaki, and Akira Onuki

(Received 25 May 2017; published 6 June 2017)

DOI: 10.1103/PhysRevE.95.069901

This Erratum corrects the data of bond-breakage susceptibility $\chi_{b}(t)$ shown in Fig. 3 in the paper. There was a technical error in the numerical procedure taken by the authors. In Fig. 1 of this Erratum, for each curve obtained from the corrected data of $\chi_{b}(t)$ (averaged over eight independent runs), its peak height is lower and it monotonically decreases to zero for $t>t_{b}^{\text {max }}$. For $T=0.56$, the equilibration time after quenching is taken on the order of $10^{6}$ in units of the time $\tau_{0}$ in Eq. (2.3), which is much longer than in the paper.

The sentence in Sec. III C, "Here, however, $\chi_{b}(t)$ begins to increase for $t>t_{b}^{\max }$, because the $\boldsymbol{B}$ particles with $\mathcal{B}_{i}=2,3, \ldots$ become appreciable at very long times (see Fig. 1)," should be deleted. However, the peak time $t_{b}^{\max }$ is little changed by the error and all the other results, including those on the structure factor $S_{b}\left(q, t_{b}^{\max }\right)$ and the correlation length $\xi_{b}\left(t_{b}^{\max }\right)$, are not affected. Thus, the conclusions presented in the paper remain unchanged.

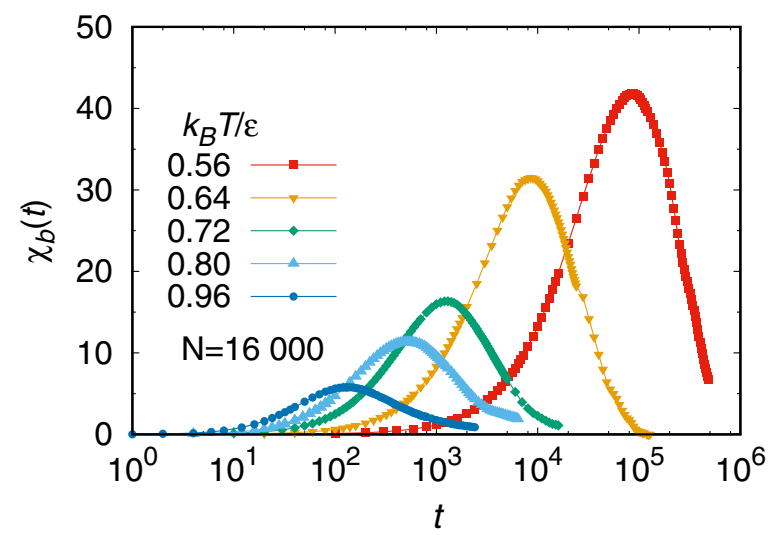

FIG. 1. Susceptibility $\chi_{b}(t)$ in Eq. (3.21) vs $t$ exhibiting a peak at $t=t_{b}^{\max }$ for $N=16000$. 